\title{
Post-surgical treatment of thyroid carcinoma in dogs with retinoic acid 9 cis improves patient outcome
}

\author{
V. Castillo ${ }^{1, *}$, P. Pessina ${ }^{2}$, P. Hall ${ }^{3}$, M.F. Cabrera Blatter ${ }^{1}$, D. Miceli ${ }^{1}$, E. Soler Arias ${ }^{1}$ and P. Vidal ${ }^{1}$ \\ ${ }^{1}$ Cat. Clin. Méd. Peq. An. and U. Endocrinología, Escuela Medicina Veterinaria, Facultad de Ciencias Veterinarias, \\ Universidad de Buenos Aires. Av.Chorroarín 280, C. Autónoma de Buenos Aires, Argentina \\ ${ }^{2}$ Laboratorio de Técnicas Nucleares, Facultad de Veterinaria, Universidad de la República, Lasplaces 1550, \\ Montevideo, Uruguay \\ ${ }^{3}$ Cat. Cirugía and U. Cirugía, Hosp., Escuela Medicina Veterinaria, Facultad de Ciencias Veterinarias, Universidad \\ de Buenos Aires. Av.Chorroarín 280, C. Autónoma de Buenos Aires, Argentina
}

\begin{abstract}
The objective of the present study was to compare the effects of isotretinoin 9-cis (RA9-cis) as a post-surgery treatment of thyroid carcinoma to a traditional treatment (doxorubicin) and no treatment. Owners who did not want their dogs to receive treatment were placed into the control group $\mathrm{A}(\mathrm{GA} ; \mathrm{n}=10)$. The remaining dogs were randomly placed into either group $\mathrm{B}(\mathrm{GB} ; \mathrm{n}=12)$ and received doxorubicin at a dose of $30 \mathrm{mg} / \mathrm{m}^{2}$ every three weeks, for six complete cycles or group $C(G C ; n=15)$ and treated with RA9-cis at a dose of $2 \mathrm{mg} / \mathrm{kg} /$ day for 6 months. The time of the recurrence was significantly shorter in the GA and $\mathrm{GB}$ compared to $\mathrm{GC}$ ( $\mathrm{P}=0.0007 ; \mathrm{P}=0.0015$ respectively), while we did not detect differences between GA and GB. The hazard ratio of recurrence between GA and GB compared to GC were 7.25 and 5.60 times shorter, respectively. We did not detect any differences between the other groups. The risk ratio of recurrence was 2.0 times higher in GA compared to GC and 2.1 times higher in GB compared to GC. The type of carcinoma had an effect on time of survival with follicular carcinomas having an increased mean survival time than follicular-compact carcinomas $(\mathrm{P}<0.0001)$ and follicular-compact carcinomas had a longer mean survival time than compact carcinomas. The interaction among treatment and type was significant, but survival time in follicular carcinomas did not differ between treatments. In follicular-compact carcinomas the survival time of GC was greater than GB $(\mathrm{P}<0.05)$, but we did not detect a difference between GA and GB. In conclusion, this study shows that the use of surgery in combination with RA9-cis treatment significantly increases survival rate and decreases the time to tumor recurrence when compared to doxorubicin treated or untreated dogs. The histological type of carcinoma interacted with treatment for time to recurrence and survival time, with more undifferentiated carcinomas having a worse prognosis than differentiated carcinomas.
\end{abstract}

Keywords: Differentiated follicular carcinoma, Retinoic acid, Thyroid carcinoma, Thyroid tumour.

\section{Introduction}

The vast majority (90\%) of thyroid tumours in dogs are thyroid carcinomas (TC), which is a frequent endocrine cancer in dogs (Barber, 2007). Thyroid carcinomas are classified according to their histological features and origin as: a) follicular thyroid carcinoma (FTC, arises from follicular cells); b) medullary thyroid carcinoma; and c) anaplastic (Kiupel et al., 2008; Meissner and Warren, 1969; Wucherer and Wilke, 2010). Follicular thyroid carcinomas are further divided into well differentiated (dFTC) follicular, dFTC follicular compact, dFTC compact, dFTC papillary, poorly differentiated, undifferentiated, or carcinosarcoma. Follicular thyroid carcinoma is the most frequently diagnosed histological variants with follicular-compact and compact the most common forms (Campos et al., 2014; Mitchell and Tjian 1989; Klein et al., 1995; Worth et al., 2005). The histological origin of dFTC tumours will influence its progression, local invasion, tumour recurrence after surgery, and median survival time (Haugen, 1999). However, the dFTC functional characteristics (iodine uptake, thyroid hormones synthesis) vary by histological origin (Verschueren et al., 1991).

The median survival time for dogs with untreated thyroid carcinoma is only three months (Worth et al., 2005), but in freely movable non-metastatic thyroid tumours, the median survival time with surgery alone has been reported to be from 7-8 months (Page, 2001; Morris and Dobson, 2002) to over 36 months (Klein et al., 1995). Campos et al. (2014) report that macro- or microscopic evidence of vascular invasion is a negative predictor of disease free-survival time in dogs after surgically excised TC.

In humans it is well known that therapeutic options for TC and prognosis after treatment will depend on the type of tumour, its iodine uptake, the vascular endothelial growth factor, and $\mathrm{Ki}-67$ expression (Capp et al., 2010; Coelho et al., 2011; Klein et al., 2000; Soh 
et al., 1997; Tallini et al., 1999); although, no similar studies have been conducted in dogs except for one study examining Ki-67 labeling, which was associated with local invasiveness (Campos et al., 2014).

Regarding TC treatment in dogs, particularly dFCT, the first and main therapeutic procedure is surgical removal of the tumour, if removal is feasible. For dFTC removal, whether or not there is invasion of adjacent tissues must be taken into account. Recurrence of the tumour after surgery is dependent on the histological subtype of the tumour. The worst prognosis is seen with the follicular-compact or compact thyroid carcinoma (Klein et al., 1995; Radinsky, 2007). After surgery ${ }^{131} \mathrm{I}$ therapy is recommended to eliminate remnant cancer tissue, but only if the tumour shows ${ }^{99 \mathrm{~m}} \mathrm{Tc}$ uptake. Radiation is not effective when ${ }^{99 \mathrm{~m}} \mathrm{Tc}$ uptake does not occur because poor uptake only occurs in poorly differentiated tumours or undifferentiated tumours (Worth et al., 2005; Turrel et al., 2006). In dFCT cases, radiation therapy has been associated with a median survival time of 28 months (Verschueren et al., 1991; Turrel et al., 2006). Despite the apparent success of surgery and radiation in the management of localized TC, up to $80 \%$ of canine patients have recurrences of TC or develop metastases during the course of their disease (Patnaik and Lieberman, 1991; Carver et al., 1995; DeLellis, 2004; Kiupel et al., 2008; Sipos et al., 2008; Wucherer et al., 2010). The limitation of radiotherapy is its high costs, the need of an appropriate infrastructure, and the legal regulations in each country. The use of chemotherapy for non-surgical TC or metastasis has had limited results. Doxorubicin has been the most frequently used drug, but it is only affective on differentiated TC tumours (Jeglum and Whereat, 1983; Ogilvie et al., 1989). Studies using cisplatine and nevirapine have reported similar results (Fineman et al., 1998; Muscella et al., 2009; Dong et al., 2013). On the other hand, chemotherapy after thyroidectomy has been reported to not provide a survival benefit (Nadeu et al., 2011; Tuohy et al., 2012).

Haugen et al. (2014) showed that thyroid tumours express both retinoic $\mathrm{X}$ receptor (RXR) and retinoic acid receptors (RAR) and their different isoforms. These authors also reported that the 9-cis form of retinoic acid (isotretinoin 9-cis, RA9-cis) inhibits thyroid tumour growth, but this action depends on the expression of the RXR or RAR isofroms. The results obtained in thyroid cell lines and few clinical studies in humans on RA9-cis treatment are promising (Coelho et al., 2011 Simon et al., 2002; Short et al., 2004; Trojanowicz et al., 2010); however, no reports of RA9-cis treatment on TCs in dogs are available. The effect of RA9-cis was also studied on cultured corticotroph cell tumour in dogs and in humans with Cushing's disease (Castillo et al., 2006; Labeur et al., 2009; Paez-Pereda et al., 2001; Pecori et al., 2012). These studies showed a reduction of the tumour growth, a reduction in the tumour size, and a reduction of the clinical signs and biochemical markers.

The objective of the present study was to compare the effects of RA9-cis as a novel post-surgery treatment of TC in dogs with the traditional treatment (doxorubicin), and a non-treatment control group. We compared treatment methods by analysing the time of recurrence of the tumour and survival time of the patient after treatment, while taking into account the TC histopathology.

\section{Animals and treatments}

\section{Materials and Methods}

This study was conducted at the School Hospital of Veterinary Medicine, Faculty of Veterinary Sciences, University of Buenos Aires, Argentina. All procedures were conducted in accordance with the regulations of the Animal Experimentation Committee (Faculty of Veterinary Sciences, University of Buenos Aires, Argentina). All dog owners gave their signed consent for the participation of their animals in this study. All dogs included in the present study were diagnosed with unilateral TC (one lobe affected) without evidence of metastases according to physical examination. The physical examination was done using ultrasonography with Doppler (vascularisation into the tumour is highly suspect of malignancy), scintigraphy in gamma camera with ${ }^{99 \mathrm{~m}} \mathrm{Tc}$ (full body scan), and scan computed tomography of neck, thorax and abdomen (Fig. 1). According to the radiotracer concentration (uptake) in the gammagraphy study, (Verschueren et al., 1991) tumours were classified as hot (high uptake compared to the contralateral lobe and the salivary glands that are inhibited), cold (low uptake with respect to the contralateral lobe and salivary glands, with normal uptake), or normal-uptake (both thyroid lobes uptake and the salivary glands uptake are equals). Dogs that presented any lymph node affected with local invasion or distal metastasis were excluded from the experiment.

\section{Histological procedures and thyroid tumour} classification

The thyroid tumour was surgically removed, fixed in formalin, paraffin-embedded, and stained with hematoxylin and eosin (HE). All HE-stained slides were reviewed by a two pathologist who were blinded to clinical reports. Thus, tumours were classified according to their histological characteristics in adenomas or carcinomas and their subtypes (Kiupel et al., 2008; Wucherer and Wilke 2010). The distinction between adenoma and carcinoma was based on histological evidence of capsular invasion, vascular invasion, or metastases. Tumour staging was performed as described previously (Owen, 1980). All dogs included in the study had tumour staging S-II$\mathrm{T} 2_{\mathrm{a} / \mathrm{b}}$-No-Mo (size between 2-5 cm, freely movable or fixed, no lymph node involvement, no evidence of 
distant metastases, or local invasion). In the present study, we only used dFTC follicular, follicular-compact, and compact tumours. This was done to analyse tumour recurrence, survival time, and their interaction with the treatments (Fig. 2). The other types were excluded by their low presentation in dogs (papillary, one case) or aggressive biological behaviour (anaplasic or poorly differentiated, one of each).

\section{Thyroid hormones evaluation}

At the moment of TC diagnosis, canine TSH and canine specific free thyroxine (fT4) were determined to evaluate

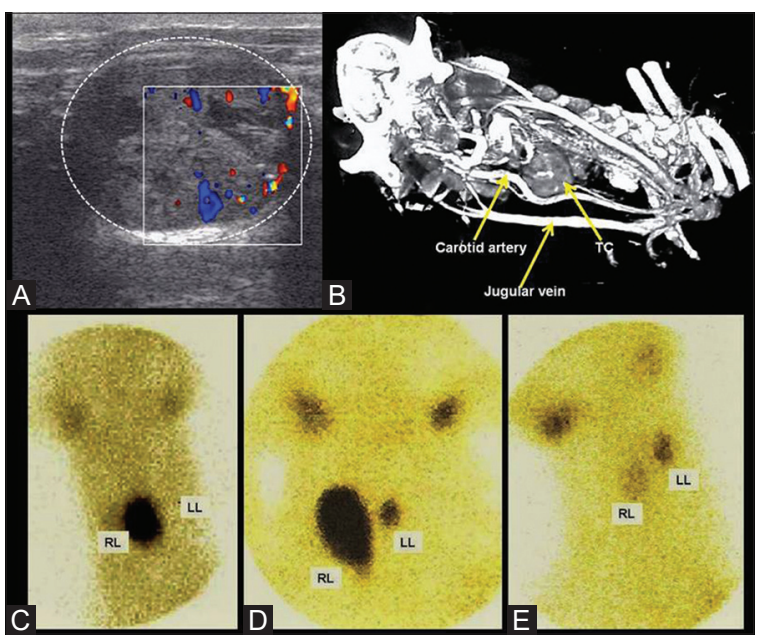

Fig. 1. Upper panel: Images of doppler ultrassonography (A), multiple slices computed tomography (B) and scintigraphy in gamma camera (C-E). Doppler ultrassonography (A) show a vascular invasion in the tumours (dotted circle show the thyroid tumour and the solid square the doppler signal). Multiple slices computed tomography (B) with extraction of the neck showing (arrow) the thyroid tumour (TC) and its relationship to the Carotid artery and Jugular vein. Bottom panel: Example of scintigraphy in dogs with follicular carcinoma and hyperthyroid (C) where the Right lobe (RL) has hot uptake and the left lobe (LL) has cold uptake; a follicular-compact carcinoma in an euthyroid dog (D) with normal uptake in both thyroid lobes, if well the size of the RL is bigger than the LL; compact carcinoma in a hypothyroid $\operatorname{dog}(\mathrm{E})$ where RL is cold (very low uptake); Add the tumour type and thyroid condition. the thyroid status. We measured canine TSH and fT4 using a chemiluminescent immunometric assay Immulite/ Immulite 1000 Canine TSH and Immulite/Immulite 1000 fT4; Siemens Healthcare Diagnostics Products Ltd., Llanberis, Gwynedd, LL55 4EL, UK) according to manufacturer's instructions.

A dog was considered to be hypothyroid when TSH was elevated $(\geq 0.4 \mathrm{ng} / \mathrm{mL})$ with $\mathrm{fT} 4$ levels within the reference range $(0.6-2.5 \mathrm{ng} / \mathrm{dL}$, subclinic hypothyroidism) or below $0.6 \mathrm{ng} / \mathrm{dL}$ (clinic hypothyroidism) as previously described (Borretti et al., 2006; Castillo et al., 2001; Ferm et al., 2009). A dog was considered to be hyperthyroid when fT4 was above $2.5 \mathrm{ng} / \mathrm{dL}$ with TSH less than $0.06 \mathrm{ng} / \mathrm{mL}$ or undetectable by the assay $(<0.03 \mathrm{ng} / \mathrm{mL})$. Both hormones were evaluated before the thyroid lobule was removed and at 90 days and 360 days post-removal.

\section{Groups performed}

Dogs that did not receive any treatment, because of the owner's decision, were placed in a control group (group A [GA]). The rest of the dogs were randomly placed into two groups. Dogs placed in group B (GB) were treated with doxorubicin (dose $30 \mathrm{mg} / \mathrm{m}^{2}$ every three weeks, six complete cycles) and dogs placed in group C were treated with RA9-cis (dose $2 \mathrm{mg} / \mathrm{kg}$ /day as enteric capsules according to the body weight of the dog for six months, as previously described (Castillo et al., 2006).

The GA $(n=10)$ included six females and four males with a median age of 7.5 (range 5-10) years; five dogs were mixed breeds and the remaining breeds consisted of: one Golden Retriever: one Labrador: one Rottweiler: one Beagle; and one Doberman. Tumours were freely movable in four dogs (S-II, T2a, No, Mo) and fixed in the six other dogs (S-II, T2b, No, Mo).

The GB $(n=12)$ included six females and six males with a median (range) age of 8.5 (4-11) years; five dogs were mixed breeds and the remaining breeds consisted of: two Beagles; one Dachshund; one Poodle; one Pekinese; one Boxer; and one Labrador. In five dogs tumours were freely movable (S-II, T2a, No, Mo) and tumours were fixed in seven dogs (S-II, T2b, No, Mo).

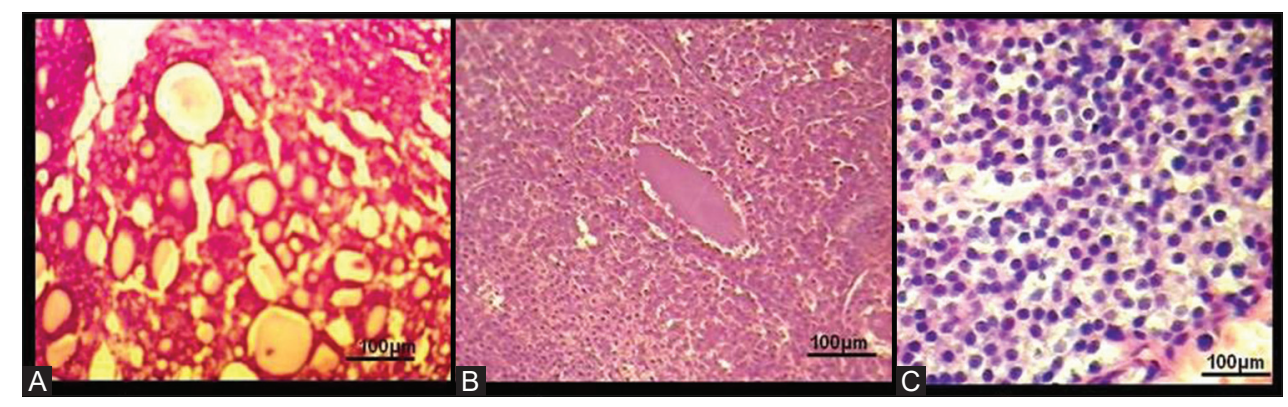

Fig. 2. Histology analysis of 3 different dFTC: A) follicular (dog was hyperthyroid); B) follicular-compact (dog was euthyroid); and C) compact (dog was hypothyroid). Bar=100 $\mu \mathrm{m}$. Staining haematoxylin and eosin. 
The GC $(\mathrm{n}=15)$ included nine females and six males with a median age of 8 (6-12) years; ten dogs were mixed breeds and the remaining dog breeds consisted of: two Boxers; one Dalmatian; one Doberman; and one Labrador. Seven tumours were freely movable (S-II, T2a, No, Mo), and the remaining eight were fixed tumours (S-II, T2b, No, Mo).

The follow-up of the cases was 540 days (1.5 years) after surgery. The first 180 days the patients received the corresponding treatment. The remaining 360 days of the follow-up no treatment was given.

All hypothyroid dogs were treated with levothyroxine (10 to $20 \mu \mathrm{g} / \mathrm{kg}$ twice a day), which continued after surgery.

\section{Statistical analysis}

The evaluation of recurrence and survival were analysed by the Long-Rank (Mantel-Cox) test, in which each step represents the recurrence or death of the individual cases. A p-value $<0.05$ was considered significant for all analyses. To calculate the risk of recurrence and the time of survival (expressed as relative risk (RR) and hazard ratio (HR), a contingent table was made to analyse differences among groups by Fisher test with a $\mathrm{P}<0.05$. The statistical software used was STATA 14 (StataCorp 4905 Lakeway Dr. College Station, TX 77845, USA). To analyse the effect of the type of tumour, the genmod procedure with the Poisson distribution was used (Statistical Analysis System version 9.0, SAS Institute, NC, USA) and the model included the effect of treatment, type of tumour (follicular, follicular-solid and solid carcinomas) and their interaction. Values are expressed as mean \pm SEM with significance at $\mathrm{P}<0.05$.

\section{Results}

\section{Thyroid status}

Before thyroid lobectomy, 32.4\% (12/37) of dogs were hypothyroid. Five of these dogs had follicularcompact tumours and the remaining seven dogs had compact carcinomas. We detected hyperthyroidism in $10.8 \%(4 / 37)$ of dogs with follicular carcinoma well differentiated. The remaining 56.8\% (21/37) of cases were euthyroid. Eight of these dogs had histological type follicular tumours, 12 had follicular-compact tumours and one had a compact carcinoma (Table 1).

Thyroid status by group was: GA $3 / 10 \quad(30 \%)$ hypothyroid; 2/10 (20\%) hyperthyroid; 5/10 (50\%) euthyroid; GB 4/12 (33.3\%) hypothyroid; $1 / 12$ (8.3\%) hyperthyroid; 7/12 (58.3\%) euthyroid, and GC 5/15 (33.3\%) hypothyroid; 1/15 (6.7\%) hyperthyroid; 9/15 (60\%) euthyroid (Fig. 3). After we removed the carcinomatous thyroid lobe from dogs with hyperthyroidism, both TSH and fT4 returned to their references range for the remainder of the study. We did not detect a change in TSH and fT4 was show in euthyroid dogs at 90 and 180 days (data no show).
Table 1. Thyroid status with respect to differentiate thyroid follicular carcinoma found in 37 dogs studied.

\begin{tabular}{lcccc}
\hline $\begin{array}{l}\text { Thyroid } \\
\text { status }\end{array}$ & \multicolumn{2}{c}{$\begin{array}{r}\text { Type of differentiate thyroid } \\
\text { folliculwar carcinoma }\end{array}$} & Total \\
\cline { 2 - 4 } & Follicular & $\begin{array}{c}\text { Follicular- } \\
\text { compact }\end{array}$ & Compact & \\
\hline $\begin{array}{l}\text { Hypothyroid } \\
(32.4 \%)\end{array}$ & -------- & 5 & 7 & 12 \\
$\begin{array}{l}\text { Hyperthyroid } \\
(10.8 \%)\end{array}$ & 4 & ------- & ------- & 4 \\
$\begin{array}{l}\text { Euthyroid } \\
(56.8 \%)\end{array}$ & 8 & 12 & 1 & 21 \\
\hline
\end{tabular}

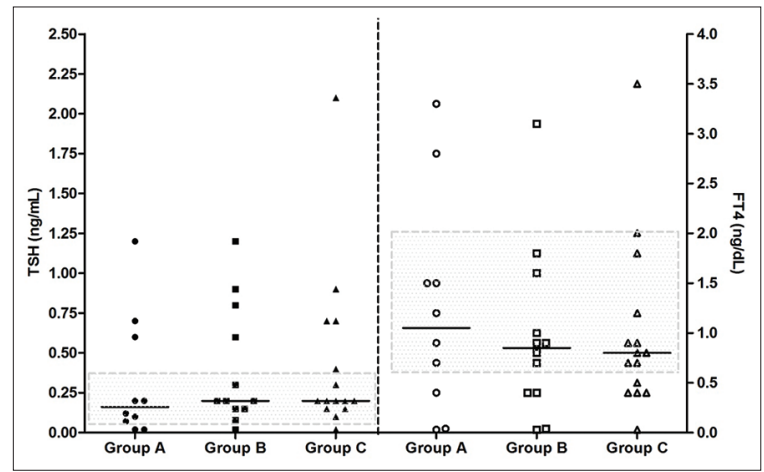

Fig. 3. Concentrations of TSH and fT4 in the control (Group A), doxorubicin (Group B), isotretinoin 9-cis (Group C) groups with follicular carcinomas. Grey areas correspond to the normal interval for the laboratory. Each dot represent one case. Horizontal lines represent the mean values.

Regardless of tumour type, thyroid carcinoma in dogs with hypothyroidism had low ${ }^{99 \mathrm{~m}} \mathrm{Tc}$ uptake (cold). Conversely, follicular tumours in dogs with hyperthyroidism ${ }^{99 \mathrm{~m}} \mathrm{Tc}$ uptake was enhanced (hot). In the euthyroid dogs with follicular-compact or compact tumours, the uptake of ${ }^{99 \mathrm{~m}} \mathrm{Tc}$ were similar in both lobes (Fig. 1).

There were not differences between freely movable or fixed tumour with respect to the ${ }^{99 \mathrm{~m}} \mathrm{Tc}$ uptake or thyroid status.

\section{Recurrence and survival time}

The time of the recurrence was significantly shorter in GA and GB compared to $\mathrm{GC}$ ( $\mathrm{P}=0.0007$ and $\mathrm{P}=0.0015$ respectively; Fig. 4). The median time for tumour recurrence for $50 \%$ of GA and GB groups was 195 days (95\% CI: $180-390)$ and 225 days (95\% CI: $210-420)$, respectively. In contrast only $13.3 \%$ of GC presented recurrence after 360 days. At the end of the experiment, recurrence was detected in $80 \%, 83.3 \%$ and $40 \%$ of the GA, GB, and GC, respectively. The HRs of tumour recurrence were 7.25 (95\% CI: 2.2-24.4) times shorter in GA compared to GC and 5.6 (95\% CI: 1.8-17.4) times shorter in GB compared to GC. We did not 
detected any difference in the HR between GA and GB (HR 1.37; 95\% CI: 0.5-3.7). The RR of recurrence was 2.0 times $(95 \% \mathrm{CI}: 1.2-4.3, \mathrm{P}=0.017)$ higher in GA when compared to the GC and 2.1 times $(95 \% \mathrm{CI}$ : 1.07-4.07, $\mathrm{P}<0.05)$ higher in GA compared to GC. We did not detect any differences between GA and GB (RR 1.08; 95\% CI: 0.78-1.5).

We also detected an increased survival time for GC compared to GA and GB (Fig. 5), but no difference between GA and GB. Median survival time was 220 days (95\% CI: 180-450) for GA and 390 days (95\% CI: 240-540) for GB. Because of the increased survival in GC, we were unable to obtain an accurate mean survival time for this group. At the end of the experiment, $20 \%$ of the dogs in GA, $33.3 \%$ of the dogs in GB, and $80 \%$ of the dogs in GC survived. The HR was 7.8 (95\% CI: 2.0-29.7) between GA and

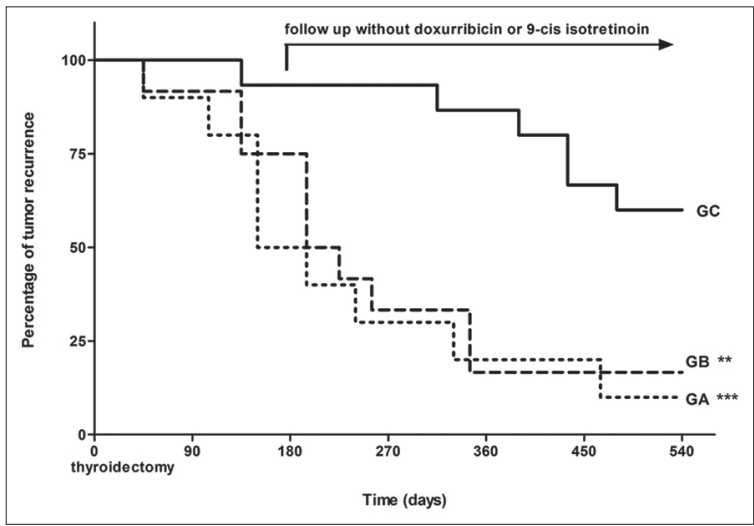

Fig. 4. Kaplan-Meier recurrence curves of thyroid tumour after surgery according to control (GA), doxorubicin (GB) and isotretinoin 9-cis (GC). Median recurrence time after surgery: GA: 195 days; GB: 225 days; GC: 360 days. $* * * \mathrm{P}=0.0007$ vs $\mathrm{GC} ; * * \mathrm{P}=0.002$ vs GC (Long-rank test).

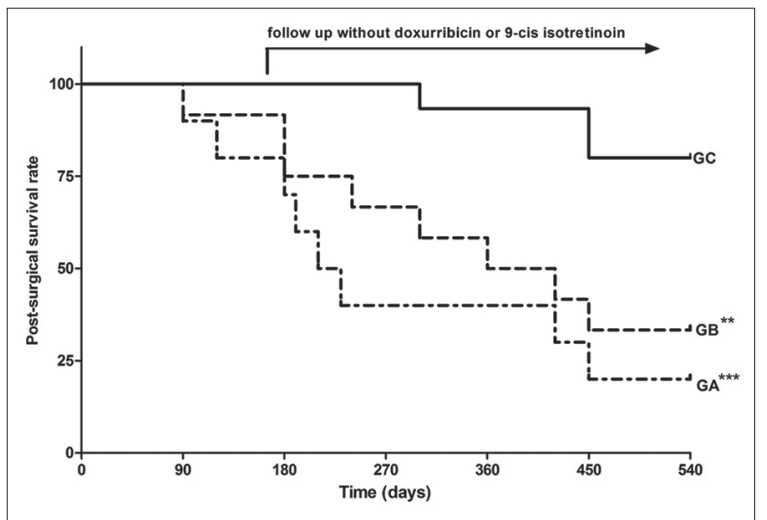

Fig. 5. Kaplan-Meier survival curves of dogs with thyroid tumour after surgery according to control (GA), doxorubicin (GB) and isotretinoin 9-cis (GC). Median survival time after surgery: GA: 220 days; GB: 390 days. In GC only 3/15 dogs had died at the end of the study (540 days). $* * * \mathrm{P}=0.0004$ vs $\mathrm{GC} ; * * \mathrm{P}=0.006$ vs GC (Long-rank test).
GB, 5.5 (95\% CI: 1.5-19.6) between GB and GC, and 1.6 (95\% CI: 0.5-4.4) between GA and GB.

\section{Interaction: type of carcinoma, treatment, recurrence and survival time}

The type of carcinoma affected time of recurrence. Follicular carcinomas had a greater length of time to recurrence than follicular-compact $(503 \pm 15 \mathrm{vs}$. $289 \pm 13$ days, $\mathrm{P}<0.0001)$. Follicular compact tumours had a greater length of time to recurrence than compact carcinomas $(215 \pm 21$ days, $\mathrm{P}<0.0001)$. Treatment affected recurrence time as $\mathrm{GC}$ had greater time of recurrence than the GB and GA (462 \pm 15 vs. $277 \pm 17$ and $268 \pm 18$ days, $\mathrm{P}<0.0001$ ), while the recurrence time of the latter two groups did not differ. The interaction between treatment and type of carcinoma was significant $(\mathrm{P}<0.001)$. We did not detect differences between treatments in follicular carcinomas, but GC had a greater time of recurrence than GB and GA in both follicularcompact and compact carcinomas (Fig. 6A and B). In $\mathrm{GC}$ the time to recurrence in follicular compact carcinomas was similar to follicular carcinomas, but time to recurrence in compact carcinomas was lower to the other two types $(\mathrm{P}<0.05)$. Freely movable $(\mathrm{T} 2 \mathrm{a})$ or fixed tumours (T2b) did not show significant differences with respect to the recurrences or survival time.

\section{Discussion}

This trail shows a novel post-surgery treatment for thyroid carcinoma in dogs. We showed that dogs treated withRA9-cis had increased survival and lower risk of

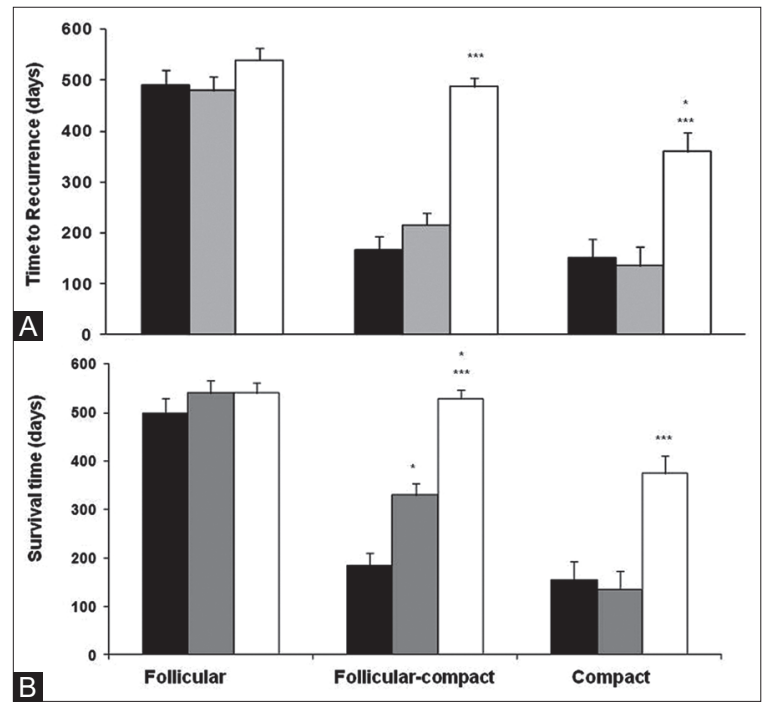

Fig. 6. Time of recurrence (A) and survival time (B) of dogs with thyroid tumour after surgery, and to the type of cancer (follicular, follicular-compact and compact). Recurrence (A): ***P $<0.001$ vs GA and GB in the same group; $* \mathrm{P}<0.05$ Compact vs Follicular-compact of the GC and $* * * \mathrm{P}<0.001$ Compact vs Follicular of the GC. Survival (B): Follicularcompact: $* * * \mathrm{P}<0.001 \mathrm{GC}$ vs $\mathrm{GA} ; * \mathrm{P}=0.026 \mathrm{GC}$ vs $\mathrm{GB}$; $* \mathrm{P}<0.05$ GB vs GA; Compact: ${ }^{* * *} \mathrm{P}<0.001 \mathrm{GC}$ vs GA and GB. Black bar: GA; Grey bar: GB; White bar: GC. 
tumour recurrence compared to dogs that received the traditional treatment (doxorubicin) or no treatment. We also showed that the treatment effect on recurrence and survival time depended on the clinical and histological type of carcinoma.This result is supported by several previous studies (Campos et al., 2014, Capp et al., 2010; Coelho et al., 2011). Follicular-compact and compact carcinomas were detected more frequently than follicular carcinomas in our study, which is in agreement with previous research (Owen, 1980; Leav et al., 1976; Théon et al., 2000). Euthyroidism status was the main feature related with the follicular-compact carcinoma, whereas hypothyroidism is associated with the compact subtype and hyperthyroidism with follicular carcinoma.

As expected, all hypothyroid patients had a low uptake of ${ }^{99 \mathrm{~m}} \mathrm{Tc}$, whereas in hyperthyroid patients that uptake was higher. There are few reports regarding the relation among thyroid carcinoma functionality and/or TC histopathology. Regarding the functional characteristics, Verschueren et al. (1991) reported that the greater the undifferentiated thyroid carcinoma profile, the less binding sites for $\mathrm{TSH}$ and ${ }^{99 \mathrm{~m} T c}$ uptake were found. Similarly, a greater expression of thyroglobulin and TSH-R was found in follicular cells within the carcinoma tissue where the remaining thyroid follicle architecture was found. This suggests that the functionality is associated to the degree of differentiation of the tumour (Pessina et al., 2014). In this study we showed that time to recurrence and survival time is affected by the histopathological subtype of the thyroid tumour (follicular, follicularcompact and compact carcinomas).

Worth et al. (2005) and Théon et al. (2000) reported that without surgery, removal, and subsequent medical treatment of TC the median survival time is three months. Although the use of chemotherapy in dogs is controversial, it improves the prognosis in non-surgical thyroid carcinoma or metastasis (Jeglum and Whereat, 1983; Ogilvie et al., 1989), but in dogs with TC treated with surgery and chemotherapy, the median survival time was not different from dogs treated with surgery alone (Nadeau and Kitchell, 2011). Since we did not detect a difference between GA and GB, the results from our study support these previous results.

Retinoic acid regulates tumour suppressor genes; therefore, it can inhibit tumour growth (Connolly et al., 2013). Retonic acid has been used successfully for the treatment of hematological cancers (acute promyelocytic leukemia), as well as a therapy and chemoprevention of solid cancers (Hansen et al., 2000; Lengfelder et al., 2005). Although there are few studies, the results of RA9-cis treatment on TC in humans are promising (Short et al., 2004; Simon et al., 2002; Trojanowicz et al., 2010). In tumour cells culture lines and human $\mathrm{TC}$, retinoic acid acts as anti-proliferative and redifferentiation agent of this disease (Coelho et al., 2005; Coelho et al., 2011; Oh et al., 2011; Trojanowicz et al., 2009). Although there has been investigated in vitro models for different tumours in dogs (de Mello Souza et al., 2010; Miyajima et al., 2006; Ohashi et al., 2006), there is only one report of using RA9-cis to treat Cushing's disease in dogs (Castillo et al., 2006). Our results clearly show that tumour recurrence is lower and survival time is longer in dogs treated with RA9cis after surgery compared with dogs that were treated with doxorubicin. Retinoic acid was shown to be less effective in dogs with compact carcinoma. These dogs had faster tumour recurrence and shorter survival time than those with other subtypes of dFTC. The reason for this difference is attributable to compact tumours being less differentiated than the other tumour types. Therefore, they have a more aggressive behavior and are more prone recurrence (Campos et al., 2014). However, in dogs with the same tumour type, RA9cis improved the time to recurrence than in those that were treated with doxorubicin and those that did not receive treatment post-surgery. Although this data is preliminary due to the low number of cases, the results of treatment with RA9-cis are promising in TC patients, mainly in those with follicular or follicular-carcinoma. Moreover, it should be noted that we did not detect any adverse effects (hepatotoxicity) related to RA9-cis treatment. This was also observed in one previous study (Ortemberg et al., 2007), and suggests that the drug is not only effective but also safe.

The most interesting finding of this study was the post-surgical survival rate of dogs treated with RA9cis and the interaction with the type of cancer. While in doxorubicin and control dogs, time to recurrence and survival time were shorter in follicular-compact carcinomas than in follicular carcinomas. In RA9-cis treated dogs we did not detect any differences between these two types of carcinomas. A similar profile was found in the comparison of follicular-compact and compact carcinomas; although, compact carcinomas presented a lower time of recurrence and survival time in RA9-cis treated dogs. The therapy response with RA9cis in both follicular-compact and compact carcinomas (e.g. more undifferentiated cells when compared with follicular carcinomas) are consistent with the function of the retinoic receptors. Therapy with RA for thyroid cancer requires intact receptor pathways in the thyroid tumour tissues and the expression of certain isoforms mainly RAR $\beta$ and RXR $\gamma$ (Haguen et al., 2004; Simon et al., 2002). We have recently shown (Pessina: personal communication), that follicular cells of both follicularcompact and compact carcinomas expressed RAR $\alpha$ and RXR in similar magnitude as healthy thyroid glands. In conclusion, this study shows that the use of surgery in combination with RA9-cis treatment significantly increase survival rate and time to tumour recurrence 
when compared to doxorubicin treated or untreated dogs. Moreover, it also demonstrates that treatment effect on recurrence and survival time depended on the histological type of the thyroid cancer.

\section{Acknowledgments}

We are grateful to Drs. Ana Meikle, Cecilia Ricart, and Nélida Gómez for the helpful support and critical reading of the manuscript. This work was supported by a grant from University of Buenos Aires-UBACyT, Argentina and University of the Republic, Laboratory of Nuclear Techniques, Uruguay.

\section{Conflict of interest}

The Author(s) declare(s) that there is no conflict of interest

\section{References}

Barber, L.G. Thyroid tumors in dogs and cats. 2007. Vet. Clin. North Am. Small Anim. Pract. 37, 756-773.

Boretti, F.S. and Reusch, C.E. 2006. Diagnostic specificity of canine thyrotropin in the diagnosis of Hypothyroidism in dogs. EJCAP. 16, 185-189.

Campos, M., Ducatelle, R., Rutteman, G., Kooistra, H.S., Duchateau, L., de Rooster, H., Peremans, K. and Daminet, S. 2014. Clinical, pathologic, and immunohistochemical prognostic factors in dogs with thyroid carcinoma. J. Vet. Intern. Med. 28, 1805-1813.

Carver, J.R., Kapatkin, A. and Patnaik, A.K. 1995. A comparison of medullary thyroid carcinoma and thyroid adenocarcinoma in dogs: a retrospective study of 38 cases. Vet. Surg. 24, 315-319.

Castillo, V., Giacomini, D., Páez-Pereda, M., Stalla, J., Labeur, M., Theodoropoulou, M., Holsboer, F., Grossman, A., Stalla, G. and Arzt, E. 2006. Retinoic Acid as a Novel Medical Therapy for Cushing's Disease in Dogs. Endocrinology 147, 4438-4444.

Castillo, V., Rodriguez, M.S., Lalia, J. 2001. Estimulación con TRH y evaluación de la respuesta de la TSH en perros. Su importancia en el diagnóstico de enfermedad tiroidea subclínica (hipotiroidismo subclínico y tiroiditis autoinmune eutiroidea). Rev. Cient-Fac. Cien. V. 11, 23-27.

Coelho, S.M., Vaisman, M. and Carvalho, D.P. 2005. Tumour re-differentiation effect of retinoic acid: a novel therapeutic approach for advanced thyroid cancer. Curr. Pharm. Des. 11, 2525-2531.

Coelho, S.M., Vaisman, F., Buescu, A., Mello, R.C., Carvalho, D.P. and Vaisman, M. 2011. Follow-up of patients treated with retinoic acid for the control of radioiodine non-responsive advanced thyroid carcinoma. Braz. J. Med. Biol. Res. 44, 73-77.

Connolly, R.M., Nguyen, N.K. and Sukumar, S. 2013. Molecular pathways: current role and future directions of the retinoic acid pathway in cancer prevention and treatment. Clin. Cancer Res. 19, 1651-1659.
De Lellis, R.A. 2004. International Agency for Research on Cancer. World Health Organization, International Academy of Pathology, International Association for the Study of Lung Cancer. Pathology and genetics of tumours of endocrine organs. Lyon: IARC Press, pp: 320.

deMelloSouza, C.H., Valli, V.E., Selting, K.A., Kiupel,M. and Kitchell, B.E. 2010. Immunohistochemical detection of retinoid receptors in tumors from 30 dogs diagnosed with cutaneous lymphoma. J. Vet. Intern. Med. 24, 1112-1127.

Dong, J.J., Zhou, Y., Liu, Y.T., Zhang, Z.W., Zhou, X.J., Wang, H.J. and Liao, L. 2013. In vitro evaluation of the therapeutic potential of nevirapine in treatment of human thyroid anaplastic carcinoma. Mol. Cell Endocrinol. 370, 113-118.

Ferm, K., Björnerfeldt, S., Karlssony, A., Andersson, G., Nachreinerz, R. and Hedhammar, A. 2009. Prevalence of diagnostic characteristics indicating canine autoimmune lymphocytic thyroiditis in giant schnauzer and hovawart dogs. J. Small Anim. Pract. 50, 176-179.

Fineman, L.S., Hamilton, T.A., de Gortari, A. and Bonney, P. 1998. Cisplatin chemotherapy for treatment of thyroid carcinoma in dogs: 13 cases. J. Am. Anim. Hops. Assoc. 34, 109-112.

Hansen, L.A., Sigman, C.C., Andreola, F., Ross, S.A., Kelloffm, G.J. and De Luca, L.M. 2000. Retinoids in chemoprevention and differentiation therapy. Carcinogenesis 21, 1271-1279.

Haugen, B.R., Larson, L.L., Pugazhenthi, U., Hays, W.R., Klopper, J.P., Kramer, C.A. and Sharma, V. 2004. Retinoic acid and retinoid X receptors are differentially expressed in thyroid cancer and thyroid carcinoma cell lines and predict response to treatment with retinoids. J. Clin. Endocrinol. Metab. 89, 272-280.

Jeglum, K.A. and Whereat, A. 1983. Chemotherapy of canine thyroid carcinoma. Comp. Cont. Educ. Pract. 5, 96-98.

Kebebew, E., Peng, M., Treseler, P.A., Clark, O.H., Duh, Q.Y., Ginzinger, D. and Miner, R. 2004. Id1 gene expression is up-regulated in hyperplastic and neoplastic thyroid tissue and regulates growth and differentiation in thyroid cancer cells. J. Clin. Endocrinol. Metab. 89, 6105-6111.

Kiupel, M., Capen, C., Miller, M. and Smedley, R. 2008. Histological classification of the endocrine system of domestic animals. In: Schulman FY, ed. WHO international histological classification of tumors of domestic animals. Washington: Armed Forces Institute of Pathology.

Klein, M.K., Powers, B.E., Withrow, S.J., Curtis, C.R., Straw, R.C., Ogilvie, G.K., Dickinson, K.L., Cooper, M.F. and Baier, M. 1995. Treatment of thyroid carcinoma in dogs by surgical resection 
alone: 20 cases (1981-1989). J. Am. Vet. Med. Assoc. 206, 1007-1009.

Labeur, M., Paez-Pereda, M., Arzt, E. and Stalla, G.K. 2009. Potential of retinoic acid derivatives for the treatment of corticotroph pituitary adenomas. Rev. Endocr. Metab. Disord. 10, 103-109.

Leav, I., Schiller, A.L., Rijnberk, A., Legg, M.A. and der Kinderen, P.J. 1976. Adenomas and carcinomas of the canine and feline thyroid. Am. J. Pathol. 83, 61-93.

Mayer, M.N. and MacDonald, V.S. 2007. External beam radiation therapy for thyroid cancer in the dog. Can. Vet. J. 48, 761-763.

Meissner, W.A. and Warren, S. 1969. Tumors of the thyroid gland. Atlas of Tumor Pathology,

Second series. Fascicle 4. Washington DC, Armed Forces Institute of Pathology.

Mitchell, M., Hurov, L.I. and Troy, G.C. 1979. Canine thyroid carcinomas: clinical occurrence, staging by means of scintiscans and therapy of 15 cases. Vet. Surg. 8, 112-118.

Miyajima, N., Watanabe, M., Ohashi, E., Mochizuki, M., Nishimura, R., Ogawa, H., Sugano, S. and Sasaki, N. 2006. Relationship between retinoic acid receptor alpha gene expression and growth-inhibitory effect of all-trans retinoic acid on canine tumor cells. J. Vet. Intern. Med. 20, 348-354.

Muscella, A., Urso, L., Calabriso, N., Vetrugno, C., Fanizzi, F.P., Storelli, C. and Marsigliante, S. 2009. Functions of epidermal growth factor receptor in cisplatin response of thyroid cells. Biochem. Pharmacol. 77, 979-992.

Nadeau, M.E. and Kitchell, B.E. 2011. Evaluation of the use of chemotherapy and other prognostic variables for surgically excised canine thyroid carcinoma with and without metastasis. Can. Vet. J. 52, 994-998.

Oh, S.W., Moon, S.H., Park Do, J., Cho, B.Y., Jung, K.C., Lee, D.S. and Chung, J.K. 2011. Combined therapy with ${ }^{131} \mathrm{I}$ and retinoic acid in Korean patients with radioiodine-refractory papillary thyroid cancer. EJNMMI. 38, 1798-1805.

Ohashi, E., Miyajima, N., Nakagawa, T., Takahashi, T., Kagechika, H., Mochizuki, M., Nishimura, R. and Sasaki, N. 2006. Retinoids induce growth inhibition and apoptosis in mast cell tumor cell lines. J. Vet. Med. Sci. 68, 797-802.

Ogilvie, G.K., Reynolds, H.A., Richardson, R.C., Withrow, S.J., Norris, A.M., Henderson, R.A., Klausner, J.S., Fowler, J.D. and McCaw, D. 1989. Phase II evaluation of doxorubicin for the treatment of various canine neoplasms. J. Am. Vet. Med. Assoc. 195, 1580-1583.

Ortemberg, L., Loiza, M., Martiarena, B., Cabrera Blatter, M.F., Ghersevich, M.C. and Castillo, V. 2007. Retinoic acid as a therapy for Cushing's disease in dogs: evaluation of liver enzymes during treatment. Slov. Vet. Res. 44, 73-81.

Owen, N. 1980. TNM classification of tumours in domestic animals World Health Organization, Geneva, pp: 51-52.

Page, R.L. 2001. Tumors of the endrocrine system. In: Withrow SJ, MacEwen EG (eds). Small Animal Clinical Oncology. $3^{\text {rd }}$ ed. W B Saunders, Philadelphia, USA, pp: 423-433.

Páez-Pereda, M., Kovalovsky, D., Hopfner, U., Theodoropoulou, M., Pagotto, U., Uhl, E., Losa, M., Stalla, J., Grübler, Y., Missale, C., Arzt, E. and Stalla, G.K. 2001. Retinoica cid prevents experimental Cushing síndrome. J. Clin. Invest. 108, 1123-1131.

Patnaik, A.K. and Lieberman, P.H. 1991. Gross, histologic, cytochemical, and immunocytochemical study of medullary thyroid carcinoma in sixteen dogs. Vet. Pathol. 28, 223-233.

Pecori, G., Ambrogio, A.G., Andrioli, M., Saguin, F., Karamouzis, I., Corsello, S.M., Scaroni, C., Arvat, E., Pontecorvi, A. and Cavagnini, F. 2012. Potential role for retinoic acid in patients with cushing's disease. J. Clin. Endocrinol. Metab. 10, 3577-3583.

Pessina, P., Castillo, V., Sartore, I., Borrego, J. and Meikle, A. 2014. Semiquantitative immunohistochemical marker staining and localization in canine thyroid carcinoma and normal thyroid gland. Vet. Comp. Oncol. Aug 1. doi: 10.1111/vco.12111.

Radlinsky, M.G. 2007. Thyroid surgery in dogs and cats. Vet. Clin. North Am. Small Anim. Pract. 37, 789-798.

Short, C.S., Suovuori, A., Cook, G., Viviany, G. and Harmer, C. 2004. A phase II study using retinoids as redifferentiation agents to increase iodine uptake in metastatic thyroid cancer. Clin. Oncol. 16, 569-574.

Simon, D., Körber, C., Krausch, M., Segering, J., Groth, P., Görges, R., Grünwald, F., MüllerGärtner, H.W., Schmutzler, C., Köhrle, J., Röher, H.D. and Reiners, C. 2002. Clinical impact of retinoids in redifferentiation therapy of advanced thyroid cancer: final results of a pilot study. EJNMI 29, 775-782.

Sipos, J.A. and Mazzaferri, E.L. 2008. Differentiated thyroid carcinoma. In: Cooper DS, ed. Medical Management of Thyroid Disease, Second ed. New York: Informa Healthcare USA, Inc. pp: 237-295.

Tallini, G., Garcia-Rostan, G., Herrero, A., Zelterman, D., Viale, G., Bosari, S. and Carcangiu, M.L. 1999. Downregulation of p27KIP1 and Ki67/ Mib1 labeling index support the classification of thyroid carcinoma into prognostically relevant categories. Am. J. Surg. Pathol. 23, 678-685.

Théon, A.P., Marks, S.L., Feldman, E.S. and Griffey, S. 
2000. Prognostic factors and patterns of treatment failure in dogs with unresectable differentiated thyroid carcinomas treated with megavoltage irradiation. J. Am. Vet. Med. Assoc. 216, 1775-1779.

Trojanowicz, B., Sekulla, C., Lorenz, K., Köhrle, J., Finke, R., Dralle, H. and Hoang-Vu, C. 2010. Proteomic approach reveals novel targets for retinoic acid-mediated therapy of thyroid carcinoma. Mol. Cell Endocrinol. 325, 110-117.

Tuohy, J.L., Worley, D.R. and Withrow, S.J. 2012. Outcome following simultaneous bilateral thyroid lobectomy for treatment of thyroid gland carcinoma in dogs: 15 cases (1994-2010). J. Am. Vet. Med. Assoc. 24, 95-103.

Turrell, J.M., McEntee, M.C., Burke, B.P. and
Page, R.L. 2006. Sodium iodide I 131 treatment of dogs with nonresectable thyroid tumors: 39 cases (1990-2003). J. Am. Vet. Med. Assoc. 229, 542-548. Verschueren, C.P., Selman, P.J., Mol, J.A., Vos, J.H., van Dijk, J.E., Sjollema, B.E. and de Vijlder, J.J. 1991. Circulating thyroglobulin measurements by homologous radioimmunoassay in dogs with thyroid carcinoma. Acta Endocrinol. (Copenh). 125, 291-298.

Worth, A.J., Zuber, R.M. and Hocking, M. 2005. Radioiodide (I131) therapy for the treatment of canine thyroid carcinoma. Aust. Vet. J. 83, 208-214.

Wucherer, K.L. and Wilke, V. 2010. Thyroid cancer in dogs: an update based on 638 cases (1995-2005). J. Am. Anim. Hops. Assoc. 46, 249-25. 